heit experimentell festgestellt ist, daß aber die Existenz eines solchen äußeren magnetokatalytischen Effektes für gewöhnliche chemische Reaktionen in gasförmiger und erst recht flüssiger Phase weder positiv noch negativ bewiesen ist. Jedenfalls spricht die Auffindung eines solchen äußeren magnetokatalytischen Effektes ausdrücklich für die Auffassung Hedvalls ${ }^{5}$, daß der von ihm entdeckte ,innere“" magnetokatalytische Effekt primär vom magnetischen Zustand der Festkörper abhängt.

Es ist uns eine angenehme Pflicht, den Herren Kollegen R. Becker-Göttingen, W. Döring-Gießen,
H. P. A. Groll-Göteborg, J. A. Hedvall-Göteborg, M. Kersten-Hanau, M. Kohler-Braunschweig und R. Ochsenfeld-Braunschweig für anregende Diskussionen zu danken. Herrn H. P. A. Groll danken wir für die Herstellung geeigneter Katalysatoren und für experimentelle Ratschläge, den Herrn H. v. Klitzing und R. Ochsenfeld für die Messung der Magnetisierungskurven. Besonderen Dank schulden wir schließlich dem Direktor der Svenska Oljeslageri AB, Göteborg, Herrn H. E. Helmerss on für die finanzielle Unterstützung, durch die diese Untersuchungen ermöglicht wurden; der Deutschen Forschungsgemeinschaft danken wir für Leihgaben an Meßinstrumenten.

\title{
Zur Thermodynamik von Flüssigkeitsmischungen verschiedener Molekülgröße
}

\author{
Mischungswärmen des Systems Benzol — Diphenyl
}

Von G. Kortüm, G. Dreesen und H.-J. Freier

Aus dem Institut für Physikalische Chemie der Universität Tübingen

(Z. Naturforschg. 8a, 546-555 [1953]; eingegangen am 6. August 1953)

\begin{abstract}
Es werden die integralen Mischungswärmen des Systems Benzol-Diphenyl bei $71,6^{\circ} \mathrm{C}$ über den ganzen Molenbruch gemessen; sie lassen sich durch die einkonstantige Gleichung für reguläre Mischungen innerhalb der Meßgenauigkeit wiedergeben. Mit Hilfe der daraus berechneten differentiellen Mischungswärmen und der aus neueren Messungen anderer Autoren entnommenen Aktivitätskoeffizienten des Benzols werden die differentiellen Zusatz-Mischungsentropien des Benzols ermittelt und mit den aus der Theorie der athermischen Mischungen berechneten Werten verglichen. Zwischen Experiment und Theorie bestehen beträchtliche Diskrepanzen, wie auch schon aus der Temperaturabhängigkeit der Mischungsenthalpien hervorgeht. Als mögliche Deutungen für diese Abweichungen werden die bei höheren Temperaturen zunehmenden Diskontinuitäten des Flüssigkeitsgitters und die ebenfalls temperaturabhängigen Orientierungseffekte in den reinen Flüssigkeiten diskutiert. Die Methodik kalorimetrischer Präzisionsmessungen bei höheren Temperaturen wird ausführlich beschrieben.
\end{abstract}

\section{Problemstellung}

$\mathrm{M}$ ischungen, bei denen sich die Komponenten ohne Enthalpieänderung mischen, bezeichnet man als athermisch. Für die thermodynamischen Mischungseffekte (bezogen auf ein Mol Mischung) gilt danach

$$
\Delta \bar{H}=0, \Delta \bar{G}=-T \Delta \bar{S} .
$$

Unterscheiden sich die Komponenten einer solchen Mischung merklich in der Größe oder der Form der Moleküle, so ist zu erwarten, worauf zuerst Gug genheim $^{1}$ hingewiesen hat, daß die Mischungs-

${ }^{1}$ E. A. Guggenheim, Trans. Faraday, Soc. 33, 151 [1937].

${ }_{2}$ Ausführliche Literaturangaben z. B. bei J. H. Hildebrand u. R. L. Scott, Solubility of Nonelectrolytes, 3. Aufl. Reinhold Publishing Corporation, New entropie vom Idealwert abweicht, so daß nach (1) auch Abweichungen vom Raoultschen Gesetz auftreten müssen. In der Folgezeit haben dann zahlreiche Autoren ${ }^{2}$ versucht, die Mischungseffekte $\Delta \bar{S}$ und $\Delta \bar{G}$ für Moleküle verschiedener Größe auf statistisch-mechanischem Wege zu berechnen. Die Mehrzahl dieser statistischen Theorien beruht auf dem Quasi-Gittermodell der Flüssigkeiten. Jedes Molekül besetzt je nach seiner Größe einen oder mehrere Plätze des Gitters, wobei sich dieses zwar nur über kleine Bereiche erstrecken soll, aber doch als genügend ausgedehnt angesehen wird, daß man

York 1950 ; E. A. Guggenheim, Mixtures, University Press Oxford 1952; G. Kortüm u. H. BuchholzMeisenheimer, Destillation u. Extraktion von Flüssigkeiten, Springer-Verlag, Heidelberg 1952. 
die Diskontinuitäten vernachlässigen kann. Die Koordinationszahl $z$ des Gitters wird also als zeitlicher Mittelwert aufgefaßt. Jeder Gitterplatz muß wahlweise von einem Molekül der Sorte l oder von einem Segment eines Moleküls der (z. B. r-polymeren) Sorte 2 besetzt werden können, ohne daß sich der makroskopische Zustand der Mischung ändert.

Streng athermische Mischungen kommen, wie das Experiment zeigt, nicht vor und sind auch auf Grund der Dispersionswechselwirkung der Moleküle grundsätzlich nicht möglich, wie vor kurzem Kuhn und Massini ${ }^{3}$ gezeigt haben. Die statistische Berechnung der Mischungseffekte an Hand des Quasigittermodells ist deshalb auch auf nichtathermische Mischungen von Molekülen verschiedener Größe ausgedehnt worden ${ }^{4}$, wobei als weiterer Parameter die Wechselwirkungsenergie der Moleküle und Segmente in Form einer Wechselwirkungskonstanten in die Rechnung eingeht. Die Theorie liefert in erster Näherung die Mischungseffekte unter der Annahme, daß die Verteilung der Moleküle vollkommen statistisch ungeordnet bleibt, in zweiter Näherung unter der Annahme, daß bestimmte gegenseitige Lagen in Form quasichemischer Gleichgewichte bevorzugt sind, wobei die Wechselwirkungsenergie gewöhnlich als klein gegenüber $k T$ angenommen wird.

Die Ergebnisse der statistischen Rechnung sind an einer Reihe von angenähert athermischen Mischungen mit Hilfe von Dampfdruckmessungen geprüft worden, insbesondere an einer Reihe von Mischungen von Alkanen verschiedener Kettenlänge ${ }^{5}$, an dem System Benzol-Diphenyl ${ }^{6}$ und an Lösungen von Polymeren ${ }^{7}$. Bei normalen Paraffinen war die Übereinstimmung zwischen Theorie und Experiment nur bei einer bestimmten Temperatur befriedigend, dagegen beobachtete man stark temperaturabhängige Mischungswärmen, so daß auch die Mischungsentropien temperaturabhängig sein müssen, was bedeutet, daß die Übereinstimmung bei

${ }^{3}$ W. Kuhn u. P. Massini, Helv. chim. Acta 33, 737 [1950].

${ }^{4}$ W. J. C. Orr, Trans. Faraday Soc. 40, 320 [1944]; E. A. Guggenheim, Proc. Roy. Soc. [London], Ser. A 183, 203, 213 [1944]; Mixtures, Oxford 1952.

5 J.H. van der Waals u. J. J. Hermans, Recueil Trav. chim. Pays-Bas 69, 949, 971 [1950]; J. H. van der Waals, ibid. 70, 101 [1951]; V. Mathot, Bull. Soc. chim. Belges 59, 111 [1950].

${ }^{6}$ H. Tompa, J. Chem. Physics 16, 292 [1948]; Mh. Chem. 83, 1356 [1952]; J. H. Baxendale, B. V. Enüstün u. J.Stern, Philos. Trans. Roy. Soc. London, anderen Temperaturen nicht mehr vorhanden sein kann. Bei verzweigten Paraffinen werden die Abweichungen von der Theorie noch wesentlich gröBer und zeigen gelegentlich sogar verschiedenes Vorzeichen (exotherme Mischungswärmen!). Wesentlich besser ist die Übereinstimmung mit der Theorie beim System Benzol-Diphenyl, doch ergaben sich auch hier Anzeichen für systematische Abweichungen, deren Gründe sich aus Dampfdruckmessungen allein nicht ermitteln ließen, die aber von den Autoren auf Differenzen der Entropiewerte gegenüber den theoretisch berechneten zurückgeführt werden. Auch bei Lösungen von Polymeren (z. B. von Polystyrol) liegen die beobachteten Mischungsentropien den idealen Werten in den meisten Fällen viel näher, als nach der Gittertheorie zu erwarten ist.

Wie diese kurze Übersicht zeigt, kann in der Thermodynamik der Mischungen von Komponenten verschiedener Molekülgröße von einer Übereinstimmung zwischen Experiment und statistischer Theorie bisher nicht gesprochen werden ${ }^{8}$. Offenbar bedarf es weiterer und möglichst genauer experimenteller Daten, wobei außer der freien Mischungsenthalpie aus Dampfdruckmessungen auch direkte kalorimetrische Messungen der Mischungsenthalpie notwendig sind. Dies geht daraus hervor, daß nach neueren Messungen von $\mathrm{E}$ bert und Mitarbeitern ${ }^{9}$ sowohl wie nach einfachen theoretischen Überlegungen ${ }^{10}$ Entropien und Mischungswärmen wesentlich empfindlicher sind gegenüber Mängeln oder unzulässigen Vereinfachungen des zur theoretischen Rechnung benutzten Modells als die freien Enthalpien. Wir haben deshalb am System Benzol-Diphenyl kalorimetrische Messungen der Mischungswärmen ausgeführt, da an diesem System sehr genaue und übereinstimmende Dampfdruckmessungen von verschiedenen Autoren vorliegen, die anscheinend die Orr-Guggenheimsche Theorie aufs beste bestätigen. Bei Zimmertemperatur ist Diphenyl nur zu etwa $35 \mathrm{Mol} \%$ in Benzol löslich, so

Ser. A 243, 169 [1951]; P.W. Allen, D. H. Everett u. M. F. Penney, Proc. Roy. Soc. [London], Ser. A 212, 149 [1952]; D. H. Everett u. M. F. Penney, ibid. 212, 164 [1952].

${ }^{7}$ Vgl. dazu H. Tompa, Mh. Chem. 83, 1356 [1952].

${ }^{8}$ Vgl. dazu auch die Diskussion von H. Tompa, Mh. Chem. 83, 1356 [1952].

${ }_{9}^{9}$ L. Ebert u. Mitarb., Mh. Chem. 80, 731 [1949]; 81, 551,562 [1950]; 82, 63 [1951]. VIII.
${ }^{10}$ J.H. Hildebrand u. R.L. Scott, l. c. ${ }^{2}$, Kap. 
daß die Prüfung der Theorie über den ganzen Konzentrationsbereich nicht möglich ist. Deshalb wurden die Messungen oberhalb des Diphenylschmelzpunktes bei etwa $71^{0} \mathrm{C}$ ausgeführt, so daß sie über den gesamten Molenbruch ausgedehnt werden konnten. Da auch Dampfdruckmessungen bei $65^{\circ}$, $70^{0}$ und $75^{0}$ vorliegen, sind so alle Daten zur Berechnung der thermodynamischen Mischungseffekte gegeben.

\section{Experimentelle Methode}

Die zu erwartenden Mischungsenthalpien beim System Benzol-Diphenyl sind sehr klein. Eine Überschlagsrechnung ergibt, daß bei Verwendung einer Substanzmenge von etwa 30-40 g die Temperaturänderungen höchstens $0,1^{0} \mathrm{C}$ betragen. Da diese jedoch nicht direkt meßbar sind, sondern aus der Temperatur-Zeit-Kurve extrapoliert werden müssen, konnten genügend genaue Resultate nur mit elektrischen Meßeinrichtungen erreicht werden. Dabei wurde das Widerstandsthermometer dem Thermoelement vorgezogen, weil dann die Meßgenauigkeit nicht von der Konstanz eines geeigneten Temperatur-Fixpunktes und damit indirekt von der Meßtemperatur abhängig wird.

Größere Schwierigkeiten als die genaue Temperaturmessung bereiten andere Umstände, die kurz besprochen werden sollen: Bei der Meßtemperatur von ca. $71^{0} \mathrm{C}$ beträgt der Dampfdruck des reinen Benzols rund 550-580 Torr. Das Kalorimeter mußte deshalb druckfest verschlossen sein, um eine Verdampfung von Benzol zu vermeiden. Weiterhin mußten beide Flüssigkeiten im Kalorimeter gasdicht voneinander abgetrennt sein, um eine vorzeitige Durchmischung über die Gasphase unmöglich zu machen. $\mathrm{Zu}$ diesem $Z$ weck wurde der eine der zu mischenden Stoffe in eine Glasampulle eingeschmolzen, die nach Erreichung der Temperaturkonstanz im Thermostaten durch einen besonderen Mechanismus zertrümmert wurde. Schließlich sollte das nicht mit Substanz angefüllte ,,leere Volumen“ des Kalorimeters so klein wie irgend möglich sein, um die beim Herstellen der Mischung verdampfenden oder kondensierenden Benzolmengen klein zu halten ${ }^{11}$.

11 Mischt man z. B. reinem Benzol von $71^{\circ} \mathrm{C}$ die gleiche molare Menge an Diphenyl zu, so beträgt die auf Grund der Dampfdruckerniedrigung auftretende Kondensationswärme des Benzols -0,091 V cal, wenn $V$ das ,leere Volumen“ des Gefäßes bedeutet. Das waren pro $\mathrm{cm}^{3}$ etwa $1 \%$ der zu erwartenden Mischungsenthalpien.

Unter Berücksichtigung dieser Voraussetzungen wurde ein Kalorimeter entwickelt, das in Abb. 1 mit nur unwesentlichen Vereinfachungen maßstäblich dargestellt ist. Die Kalorimeterbombe befindet sich in einem geräumigen Isoliermantel, in dem sie durch vier punktförmige Stützen aus Kunststoff befestigt ist; sie besteht aus vernickeltem Messing. Nach Abzug der Einbauteile beträgt das Innenvolumen rund $52 \mathrm{~cm}^{3}$. Bei gespannter Zertrümmerungsvorrichtung ist die untere Hälfte des Gefäßes frei und kann zur Aufnahme verhältnismäßig großer Ampullen mit einem

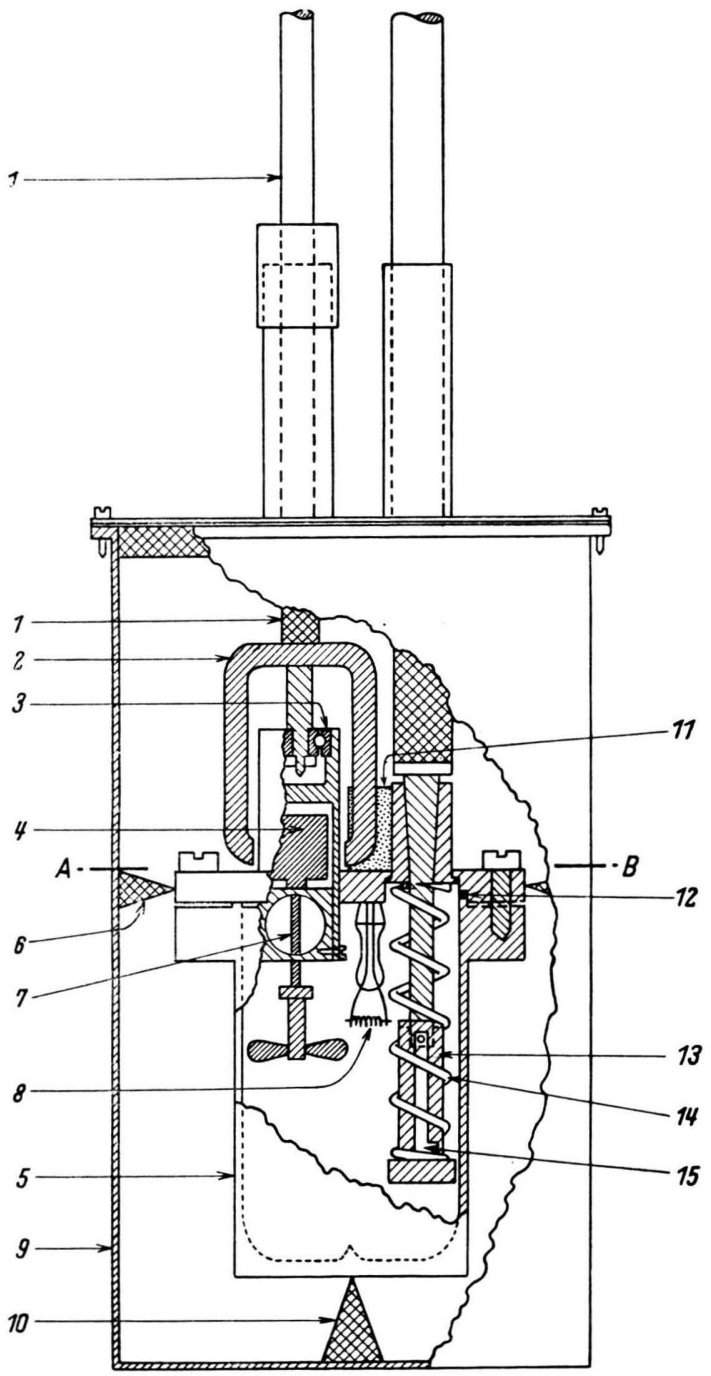

Abb. 1. Kalorimeter (Maßstab $2: 3$ ).

1 Antriebsachse

2 Magnet

3 Kugellager

4 Magnetkern

5 Kalorimetergefäß

6 Haltering aus Kunststoff

7 Rührachse

8 Heizwendel
9 Isoliermantel 10 Kunststoffspitze

11 Schliffhülsen für Heizung u. Zertrümmerer

12 Bleidichtung

13 Schlagbolzen

14 Feder

15 Bajonettverschluß 
Maximalinhalt von $17-18 \mathrm{~cm}^{3}$ dienen. Die Spitze am Boden des Gefäßes ist zur vollständigen Zertrümmerung der Ampulle erforderlich. Auf der Außenwand des Kalorimetergefäßes ist in der ganzen Länge ein Gewinde aufgeschnitten, in dessen Rillen der Widerstandsdraht untergebracht ist. Es wurden $19 \mathrm{~m}$ lackisolierter Kupferdraht von $0,08 \mathrm{~mm} \varnothing$ in bifilarer Wicklung verwendet. Vor dem Aufwickeln des Drahtes wurde das Gefäß dreimal in sehr dünner Schicht lackiert. Über den Draht wurde nochmals eine relativ dicke Lackschicht aufgetragen. Auf diese Weise wurde bei bester elektrischer Isolation ein sehr guter Wärmekontakt zwischen Kalorimetergefä $B$ und Widerstandsdraht erreicht. Aus Kapazitätsmessungen konnte die Dicke der Lackschicht zwischen Draht und Gefäß zu etwa $1 / 100 \mathrm{~mm}$ bestimmt werden.

Der Deckel des Kalorimetergefäßes trägt den Rührer, die Zertrümmerungsvorrichtung und die Heizvorrichtung. Er wird mit dem Gefä $\beta$ verschraubt und mit einem Bleiring abgedichtet. Weiterhin ist im Deckel eine mit Blei abgedichtete Schraube vorhanden, die zum Einfüllen der Substanzen dient.

Um einen absolut gasdichten und reibungsarmen Rührantrieb zu erreichen, wurde das von Kontaktthermometern her bekannte Prinzip angewandt. Der aus Messing gefertigte Rührer ist über eine $\mathrm{V}_{2} \mathrm{~A}$-Stahlachse mit einem entsprechend geformten Magnetkern aus $\mathrm{V}_{2} \mathbf{A}$-Stahl verbunden. Das Ganze ist zusammen mit einem Lagerblock in einem nach oben verschlossenen Rohr untergebracht, über das von außen ein Hufeisenmagnet aufgesetzt ist. Dieser läuft in einem kleinen Kugellager und wird durch eine Kunststoffachse angetrieben. Die Tourenzahl betrug $750 \mathrm{U} / \mathrm{min}$.

Die Zertrümmerungsvorrichtung ist in Abb. 1 in entspanntem Zustand eingezeichnet. Der Schlagbolzen wird im gespannten Zustand durch einen Bajonettverschlu $ß$ gehalten und durch Drehung der Achse freigegeben. Die Zertrümmerungsvorrichtung ist ganz aus $\mathrm{V}_{2} \mathbf{A}$-Stahl hergestellt. Die in der Feder aufgespeicherte Energie ist wegen der konstanten geometrischen Verhältnisse stets die gleiche. Sie wurde experimentell zu $0,24_{3}$ und $0,25_{8}$ cal bestimmt. Für die Berechnung der Meßergebnisse wurde ein Wert von 0,25 cal zugrunde gelegt.

Zur jeweiligen Bestimmung der Wärmekapazität des Kalorimeters (einschließlich Füllung) wurde in das Gefäß eine kleine elektrische Innenheizung eingebaut. Sie besteht aus einem Glasschliff mit eingeschmolzenen Platindrähten, an die eine $0,05 \mathrm{~mm}$ starke Wolframdrahtwendel mit einem Widerstand von ca. 19 Ohm angelötet ist. Der mit Silikonfett gedichtete Schliff wird durch den im Gefäß herrschenden Überdruck von selbst gehalten.

Die mit einem einfachen Thermostaten bei $70^{\circ} \mathrm{C}$ erreichbare Temperaturkonstanz dürfte kaum besser als $\pm 0,01^{\circ} \mathrm{C}$ sein. Aus diesem Grunde wurde ein Doppelthermostat gebaut, der aus zwei unabhängig geregelten, ineinander geschachtelten Einheiten besteht. In jedem der beiden Thermostaten befindet sich ein Tauchsieder und ein Kontaktthermometer. Im äußeren Thermostaten sind wegen seines großen Fassungsvermögens und seiner ungünstigen Form zwei gegen- sinnig wirkende Rührer eingesetzt. Weiterhin ist eine etwa $2 \mathrm{~m}$ lange Kühlschlange eingebaut. Rührer, Kontaktthermometer (Teilung in ${ }^{1} / 5^{0} \mathrm{C}$ ) und Tauchsieder des inneren Thermostaten sind auf engstem Raum vereinigt.

Um im inneren Thermostaten die gewünschte Temperaturkonstanz zu erreichen, müssen die Heizleistungen, Regeltemperaturen und Rührgeschwindigkeiten der beiden Thermostaten sehr genau aufeinander abgestimmt werden. Bei guter Einregulierung des Thermostaten war für den inneren Teil eine Heizleistung von nur 3-5 Watt erforderlich. Die Temperaturkonstanz betrug $\pm 2 \cdot 10^{-3}{ }^{0} \mathrm{C}$. Sie war begrenzt durch die Regelgenauigkeit des Kontaktthermometers und wurde durch ein Beckmannthermometer kontrolliert.

Das Kalorimetergefä $\beta$ mit Isoliermantel wurde in den Thermostaten eingebaut. Obwohl dessen Temperaturkonstanz nur $2 \cdot 10^{-3}{ }^{0} \mathrm{C}$ betrug, bewirkte die starke Pufferung durch den Isoliermantel, daß die Temperatur im Kalorimeter nur noch Schwankungen von ca. $0,5 \cdot 10^{-3}{ }^{0} \mathrm{C}$ zeigte. Die Halbwertszeit des Kalorimeters, also die Zeit, die zum halben Ausgleich einer beliebigen Temperaturdifferenz gegenüber der Umgebung nötig war, ergab sich zu etwa 25 min.

Besonderer Wert wurde auf die einwandfreie Installation der elektrischen Leitungen gelegt. Zur Temperaturmessung diente als Meßbrücke der Kompensationsapparat von $O$. Wolff. Er wurde mit einem $2 \mathrm{~V}$-Akkumulator gespeist, dessen Spannungskonstanz ständig überwacht wurde. Als Galvanometer diente ein Instrument von $\mathrm{Kipp}$ und $\mathrm{Z}$ onen mit höchster Empfindlichkeit. Im Gebrauch war das Instrument jedoch soweit geshuntet, daß einem Ausschlag von $1 \mathrm{~mm}$ eine Widerstandsänderung von etwa $5 \cdot 10^{-4}$ Ohm, bzw. eine Temperaturänderung von etwa $2 \cdot 10^{-4}{ }^{0} \mathrm{C}$ entsprach (Skala rund 2,50 m). Eine noch größere Empfindlichkeit war nicht sinnvoll, da hierdurch lediglich der Einfluß der Thermostatenschwankungen vergröbert worden wäre.

Widerstandsänderungen von $5 \cdot 10^{-4}$ Ohm ließen sich allerdings an der Meßbrücke nicht mehr abgleichen, auch dann nicht, wenn Widerstände parallel geschaltet wurden. Hieran sind in erster Linie die undefinierten Übergangswiderstände schuld, doch können sich auch Thermokräfte, Feuchtigkeit und Temperaturschwankungen (z. B. beim Berühren) sehr störend bemerkbar machen. Aus diesem Grunde wurde mit der Ausschlagsmethode gearbeitet, nachdem der Widerstand in der Brücke vor der Messung weitgehend abgeglichen war.

Da beim Berühren der Meßbrücke unkontrollierbare Sprünge des Galvanometerausschlages auftreten konnten, durfte die Einstellung während der ganzen Messung nicht geändert werden. Zudem wurde die Temperatur im Raum konstant gehalten und sämtliche Kontakte in Watte eingepackt. Anfänglich beobachtete Kriechströme konnten durch Aufstellen des Galvanometers auf Trolitulklötzen beseitigt werden. Alle Anschlüsse wurden mehrere Stunden vor der Messung hergestellt, da sich unmittelbar nach dem Anklemmen Thermokräfte beobachten ließen, die nach einiger Zeit verschwanden. Die Heizleistung des Wi- 
derstandsthermometers war verschwindend gering. Sie ergibt sich zu ca. 9,5 $10^{-5} \mathrm{cal} / \mathrm{sec}$.

Die Schaltung der elektrischen Innenheizung ist in Abb. 2 dargestellt. Die Heizleistung wurde aus Stromstärke, Heizwiderstand und Zeit berechnet. Der wesentliche Teil der Schaltung ist zur Stromstärkemessung bestimmt, die aus dem Spannungsabfall am Widerstand $R_{3}$ berechnet wurde. In der Abbildung sind $\mathrm{K}$ der Kompensationsapparat von Wolff, $\mathbf{S}$ ein eingebauter Umschalter, $\mathbf{R}_{2}$ ein Kurbelwiderstand spezieller Bauart, $N$ das Normalelement, $R_{1}$ ein Stöpselwiderstand zum Einstellen des richtigen Spannungsabfalls an $R_{2}(1,49999 \mathrm{~V}$ an $14999,9 \mathrm{Ohm})$ und $R_{5}$ die Heizung. Als Vorschaltwiderstand $R_{3}$ wurden zwei parallel geschaltete Präzisionswiderstände von $10 \mathrm{Ohm}$ benutzt. $\mathrm{R}_{4}$ ist der Widerstand der Zuleitungskabel zur Heizung (ca. 0,3 Ohm).

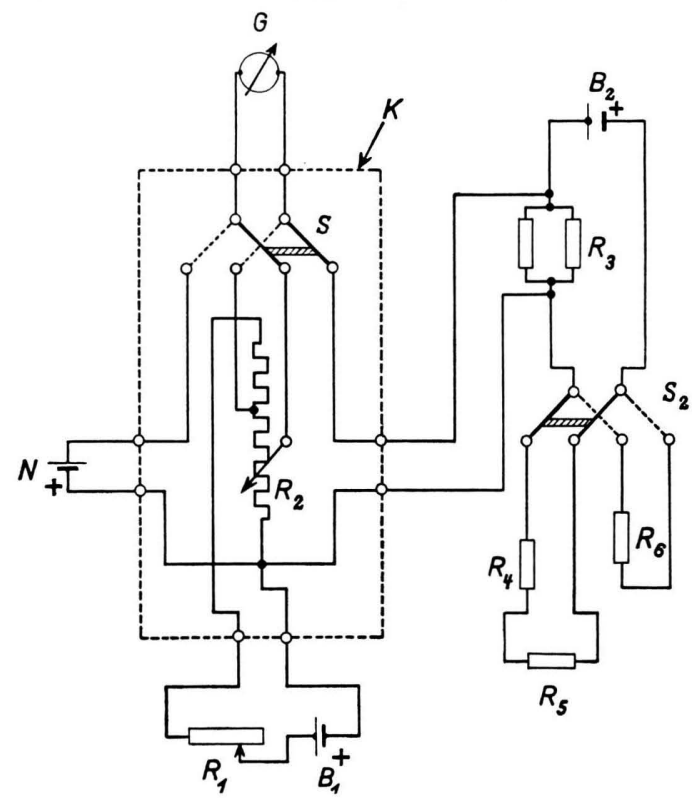

Abb. 2. Schaltung der Eichheizung.

Der Zweipolumschalter $\mathbf{S}_{\mathbf{2}}$ ermöglicht das Einschalten des Ersatzwiderstandes $\mathbf{R}_{6}$, der auf den Widerstandswert von $R_{4}+R_{5}$ eingestellt wurde. $R_{6}$ hat die Aufgabe, den Akkumulator $\mathbf{B}_{2}$ über mindestens eine Stunde vor Gebrauch der Heizung zu belasten. Dadurch wurde ein Absinken der Akkumulatorspannung während der Messung vermieden. Mit der beschriebenen Anordnung ist es möglich, Stromstärken bequem mit der Genauigkeit zu messen, die durch den Kompensationsapparat gegeben ist $\left(0,1^{0} / 00\right)$. Diese Genauigkeit wurde hier jedoch nicht erreicht, da der Kompensationsapparat für die Dauer der Versuche zur Widerstandsmessung benutzt werden mußte. Die Messung der Heizstromstärke wurde deshalb vor und nach der eigentlichen Messung ausgeführt. Aus den beiden Werten, die mindestens auf $0,5 \%$ übereinstimmten, wurde das Mittel genommen.

Die Summe der Widerstände $R_{4}+R_{5}$ wurde vor und nach der Messung - jedoch bei der Meßtempera- tur - bestimmt. Die Werte waren mindestens auf $0,2^{0} / 00$ konstant. Der Widerstand $\mathbf{R}_{\mathbf{4}}$ wurde gelegentlich gesondert bestimmt, indem die Heizspirale durch ein Stückchen Kupferdraht ersetzt wurde.

Durch die gleichzeitige Benutzung des Kompensationsapparates zur Spannungs- und Widerstandsmessung entstanden keine Schwierigkeiten. Selbstverständlich wurden auch alle für die Spannungsmessung benutzten Leitungen längere Zeit vor Beginn der Messung verlegt und die entsprechenden Kontakte in Watte eingepackt.

\section{Reinigung der Substanzen}

\section{Benzol}

Als Ausgangsprodukt diente Benzol p. a. von Merck. Zur weiteren Reinigung wurde es zweimal einige Stunden mit 80-proz. Schwefelsäure auf der Maschine geschüttelt, danach wurde mehrmals mit Wasser und verdünnter Natronlauge und abschlieBend mit frisch entgaster Tierkohle geschüttelt (jeweils wieder einige Stunden). Anschließend wurde über Natrium getrocknet und nach einer einfachen Destillation an der Jantzen-Kolonne fraktioniert. Zur Verwendung kam eine Mittelfraktion von etwa $60 \%$ der eingesetzten Menge. Sie war so bemessen, daß sämtliche Versuche mit ihr ausgeführt werden konnten.

Der Brechungsindex des reinen Benzols war

$$
n_{\mathrm{D}}^{25}=1,49798 \pm 0,00003 .
$$

Zum Trocknen des Benzols wurde eine Umlaufapparatur nach Schupp und Mecke $\mathrm{M}^{12}$ verwandt. Die Vorratskugel hatte ein Volumen von etwa $20 \mathrm{~cm}^{3}$, so daß die Umlaufgeschwindigkeit sehr groß war. Um das Benzol unter weitgehender Vermeidung von Luftzutritt in die Meßkölbchen füllen zu können, wurde am Auslaufrohr eine Schliffhülse (NS 7 ) mit eingesetzter Kapillare angeschmolzen.

\section{Diphenyl}

Als Ausgangsprodukt diente Diphenyl, rein, von Merck. Es wurde zunächst aus reinstem Alkohol umkristallisiert, danach bei Wasserstrahlvakuum destilliert, nochmals aus Alkohol und dann aus reinstem Cyclohexan umkristallisiert und zum Schluß wieder destilliert. Die Trennwirkung ist bei einer gut geführten, langsamen Destillation wesentlich größer als bei der Sublimation. Zur Reinheitskontrolle wurde das halbe Erstarrungsintervall an einer Menge von jeweils einigen Gramm bestimmt. Es war beim Ausgangsprodukt etwa 0,1 bis $0,15^{\circ} \mathrm{C}$ und beim Endprodukt 0,02 bis $0,03^{0} \mathrm{C}$.

\section{Ausführung der Messungen}

Eine der beiden Substanzen wird in eine Ampulle eingeschmolzen und in das Kalorimetergefäß gebracht. Danach wird der Deckel aufgeschraubt und die andere Substanz durch die Einfüllöffnung zugegeben. Für Benzol wird hierzu eine Wägebürette benutzt. Wegen

12 R.L. Schupp, u. R. Mecke, Z. Elektrochem.52, $59[1948]$. 
seiner geringen Flüchtigkeit kann Diphenyl in einem kleinen Kölbchen eingewogen und in geschmolzenem Zustand eingefüllt werden. Nach der Füllung wird das Kalorimeter im Isoliermantel befestigt und im Thermostaten justiert.

Wegen der guten thermischen Isolierung des Kalorimeters sind lange Heizzeiten nötig, um das Kalorimeter auf die Thermostatentemperatur zu bringen; es war deshalb zweckmäßig, die Versuche bereits abends zu beginnen. Am nächsten Morgen wird der Thermostat genau einreguliert und der Kalorimeterrührer, das Widerstandsthermometer und die ,Ersatzheizung" $R_{a}$ eingeschaltet.

Am Gang des Galvanometerausschlages läßt sich abschätzen, wie weit die Kalorimetertemperatur noch von der Thermostatentemperatur entfernt ist. Man wartet einen Zeitpunkt ab, zu dem bei kurzfristiger Einschaltung der Heizung noch keine Überhitzung zu befürchten ist und mißt die Heizstromstärke. Danach wird die Meßbrücke wieder auf Widerstandsmessung zurückgeschaltet. Nach einer kurzen Wartezeit kann mit der Aufnahme der Vorperiode begonnen werden. Ist der Galvanometergang nicht größer als $1 \mathrm{~mm} / \mathrm{min}$, das sind etwa $2 \cdot 10^{-4}{ }^{0} \mathrm{C}$, so kann die Ampulle zertrümmert werden. Um sicher zu gehen, daß dabei der Rührer nicht durch Splitter der Ampulle gestoppt wurde, wird er abgeschaltet und ohne Änderung der Tourenzahl sofort wieder eingeschaltet. Die Galvanometerausschläge der Hauptperiode werden in den ersten 15-20 min von $30 \mathrm{zu} 30 \mathrm{sec}$ abgelesen, danach von Minute zu Minute. Nach ca. 30 min wird die Innenheizung eingeschaltet.

Die Heizzeit richtet sich nach dem zuvor beobachteten Effekt und schwankt in der Regel zwischen 3 und 5 min. Schließlich wird die Nachperiode in der gleichen Weise wie die Hauptperiode aufgenommen.

Zum Schluß wird die Heizstromstärke nochmals gemessen, ebenso die Summe der Widerstände $\mathbf{R}_{4}+\mathbf{R}_{5}$.

\section{Auswertung der Messungen}

In Abb. 3 ist der Temperatur-Zeit-Gang bzw. die Kurve des Galvanometerausschlages idealisiert dargestellt. Sie setzt sich aus folgenden Teilkurven zusammen: der Vorperiode $\lambda_{1}$, der Mischungsperiode $\lambda_{2}$, der Hauptperiode $\lambda_{3}$, der Heizperiode $\lambda_{4}$ und der Nachperiode $\lambda_{5}$. Während $\lambda_{1}, \lambda_{3}$ und $\lambda_{5}$ einfache Exponentialfunktionen von der bekannten Form

$$
k t=\ln \frac{T_{1}-T_{0}}{T-T_{0}}
$$

sind, worin $k$ eine von der Isolation und der Wärmekapazität des Kalorimeters abhängige Konstante darstellt, ist $\lambda_{4}$ mit einer linearen Funktion überlagert, die so stark überwiegt, daß die Kurve in der Abbildung nicht von einer Geraden zu unterscheiden ist. $\lambda_{2}$ ist vom gleichen Typ wie $\lambda_{4}$. Ist $t_{1}$ der Zeitpunkt, an dem die Ampulle zertrümmert wird, so ergibt die Extrapolation von $\lambda_{1}$ und $\lambda_{3}$ auf $t_{1}$ den der auftretenden Mischungswärme zugeordneten Galvanometerausschlag $\Delta_{1}=x_{1}{ }^{\prime}-x_{1}$. Zur Extrapolation wurde zunächst die Nullgerade, d. h. also der der Thermostatentemperatur entsprechende Galvanometerausschlag $x_{0}$ ermittelt. Dazu trägt man $\lambda_{3}$ logarithmisch auf und variiert den geschätzten Nullwert so lange, bis sich keine systematischen Krümmungen der logarithmischen Kurve mehr erkennen lassen. Bei einiger Übung nimmt das nicht allzuviel Zeit in Anspruch. Zur Kontrolle bezieht man die Werte $\log \lambda_{1}$ auf dieselbe Nullgerade. Auch hier dürfen sich keine systematischen Krümmungen ergeben. Die gefundenen Geraden ergeben unmittelbar $\Delta_{1}$. Im Gegensatz dazu ist $\Delta_{2}=x_{2}{ }^{\prime}-x_{2}$ nicht so ohne weiteres zu bestimmen, da der Heizvorgang schon aus Gründen der genauen Zeitmessung langsam verläuft, und somit eine exakte „Bezugszeit" nicht von vornherein gegeben ist. Es läßt sich nun rechnerisch nachweisen, daß das Zeitmittel $t_{2}$ aus Beginn $t_{3}$ und Ende $t_{3}{ }^{\prime}$ der Heizperiode

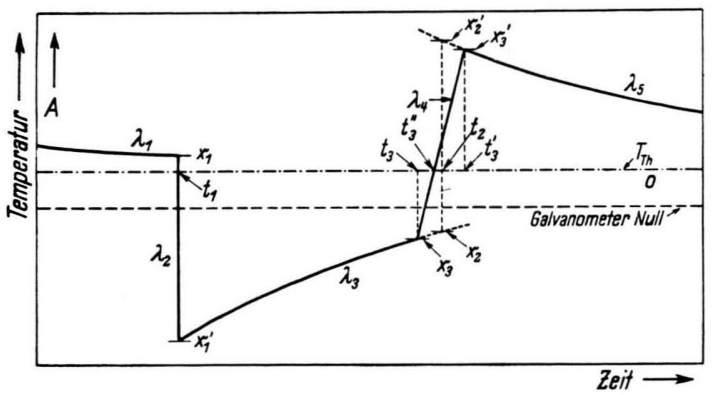

Abb. 3. Idealisierte Temperatur-Zeit-Kurve des Kalorimeters, $T_{\mathrm{Th}}=$ Temperatur des Thermostaten.

mit ausreichender Genauigkeit als Bezugszeit verwendet werden kann. Mithin kann man $\lambda_{3}$ und $\lambda_{5}$ in logarithmischer Auftragung auf $t_{2}$ extrapolieren und erhält die Differenz $\Delta_{2}$ des Galvanometerausschlages, die der Heizenergie entspricht.

Die durch Extrapolation gewonnenen Werte $\Delta_{1}$ und $\Delta_{2}$ werden mit der Eichkurve des Galvanometerausschlages korrigiert. Das Verhältnis $A^{*}{ }_{1}$ $\mathrm{zu} \Delta^{*}{ }_{2}$ ist dann gleich dem Verhältnis aus unkorrigierter Mischungsenthalpie $\Delta H^{\prime \prime}$ und Heizenergie. Die wahre Mischungsenthalpie $\Delta H^{\prime}$ erhält man aus dem Meßwert $\Delta H^{\prime \prime}$, der potentiellen Energie des Zertrümmerers $W_{z}$ und der Verdampfungskorrektur $W_{\mathrm{v}}$ :

$$
\Delta H^{\prime}=\Delta H^{\prime \prime}+W_{\mathrm{z}}-W_{\mathrm{v}} ;
$$

$W_{z}$ wird mit 0,25 cal eingesetzt. 
Unter Zugrundelegung des idealen Gasgesetzes und unter der Annahme, daß das System BenzolDiphenyl sich angenähert ideal verhält, ergibt sich $W_{\mathrm{v}} \mathrm{zu}$ :

$$
W_{\mathrm{v}}=n L_{\mathrm{v}}=\frac{p \mathrm{I}^{\mathrm{v}}}{R T} L_{\mathrm{v}}=\frac{L_{\mathrm{v}}}{R T} V x_{1} p_{01} .
$$

Hierin sind :

$n$ Menge des verdampfenden Benzols in Mol, $L_{\mathrm{v}}$ molare Verdampfungswärme des Benzols, $p=$ $x_{1} p_{01}=$ Dampfdruck des Benzols über der Mischung, $V$ leerer Raum des Kalorimeters.

$W_{\mathrm{v}}$ gilt hier für den Fall, daß vor Herstellen der Mischung kein Benzoldampf im leeren Raum des Kalorimeters vorhanden war, d. h. also, daß sich das Benzol in der Ampulle befand. Für den umgekehrten Fall muß man die Differenz $n^{\prime \prime}-n^{\prime}$ aus der Menge $n^{\prime \prime}$ des über der Mischung und der Menge $n^{\prime}$ des über dem reinen Benzol vorhandenen Dampfes in Rechnung setzen:

$$
\begin{aligned}
W_{\mathrm{v}} & =\left(n^{\prime \prime}-n^{\prime}\right) L_{\mathrm{v}} \\
& =\frac{V L_{\mathrm{v}}}{R T}\left(p^{\prime \prime}-p^{\prime}\right)=-\frac{L_{\mathrm{v}}}{R T} V x_{2} p_{01} ;
\end{aligned}
$$

$L_{\mathrm{v}}$ wurde aus den Werten der Verdampfungsenthalpie $L_{\mathrm{p}}$ von Fiock ${ }^{13}$ berechnet.

Aus den Werten: $L_{\mathrm{p}}=7,487 \mathrm{kcal} / \mathrm{Mol}$ bei $70^{\circ} \mathrm{C}$ und $L_{\mathrm{p}}=7,349 \mathrm{kcal} / \mathrm{Mol}$ bei $80^{\circ} \mathrm{C}$

wurde durch Interpolation und Verminderung um $R T$ für $71,6^{\circ} \mathrm{C}$ der Wert

$$
L_{\mathrm{v}}=6,77_{9} \mathrm{kcal} / \mathrm{Mol}
$$

erhalten. Daraus errechnet sich unter Benutzung von $p_{01}=582,2$ Torr der Wert:

$$
\frac{L_{\mathrm{v}}}{R T} p_{01}=0,183 \mathrm{cal} / \mathrm{cm}^{3} \quad \text { für } 71,6^{\circ} \mathrm{C},
$$

der der Berechnung der Korrekturen zugrunde gelegt wurde.

Schließlich läßt sich aus der korrigierten Mischungsenthalpie $\Delta H^{\prime}$ mit Hilfe der Einwaagen die gesuchte molare Mischungsenthalpie $\Delta \bar{H}$ berechnen. Es gilt:

$$
\Delta \bar{H}=\frac{\Delta H^{\prime}}{n_{1}+n_{2}} .
$$

\section{Fehlerdiskussion}

Wie bereits erwähnt wurde, ist bei der Extrapolation zunächst die Größe $x_{0}$ (Thermostatentem-

${ }^{13}$ E.F. Fiock, D.C. Ginnings u. W.B. Holton, Bur. Standards J. Res. 6, 881 [1932]. peratur) aus den Galvanometerausschlägen zu eliminieren. Die unsystematischen Streuungen des Galvanometerausschlages (hervorgerufen durch die Temperaturschwankungen des Thermostaten) erschweren diese Bestimmung, wirken sich allerdings nicht übertrieben stark aus, weil sie sich als unsystematische Streuungen in den logarithmischen Geraden wiederfinden. Von wesentlich größerem Einfluß sind demgegenüber die beobachteten systematischen Abweichungen des Galvanometerausschlages von der theoretischen Kurve.

Da die theoretische Kurve nur dann gelten kann, wenn im ganzen Kalorimeter ständig Temperaturgleichheit herrscht, lassen sich charakteristische Abweichungen erkennen, wenn im Inneren des Kalorimeters plötzliche Temperaturdifferenzen auftreten, d. h. also beim Mischen und Heizen. Erst nach einer Wartezeit von etwa 6-8 min ist der Temperaturausgleich im Kalorimeter soweit hergestellt, daß die experimentellen Kurven die theoretische Kurve innerhalb der beobachteten Schwankungen von \pm 2 bis $3 \mathrm{~mm}$ Galvanometerausschlag erreichen. Die realen Kurven $\lambda$ des Galvanometerausschlages sind in Abb. 4 (ausgezogen) der idealen

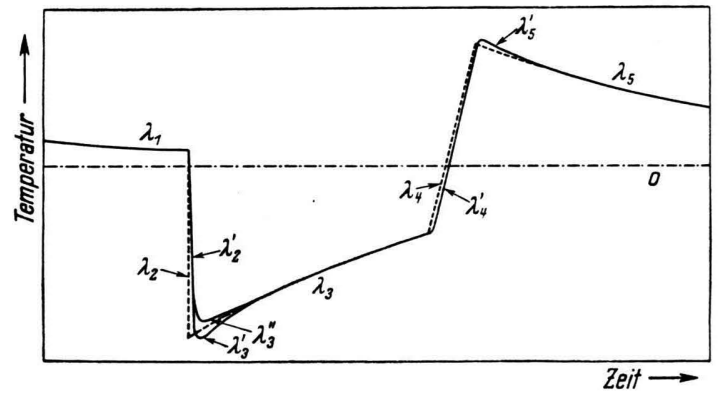

Abb. 4. Ideale und reale Temperatur-Zeit-Kurve des Kalorimeters.

Kurve $\lambda$ (gestrichelt) gegenübergestellt. Für $\lambda_{1}$ sind reale und ideale Kurve identisch; für $\lambda_{2}$ sind die Abweichungen ohne Bedeutung, da auf $t_{1}$ zurückextrapoliert wird; das gleiche gilt für $\lambda_{4}$. Für die Hauptperiode $\lambda_{3}$ sind die systematischen Abweichungen insofern bei der Auswertung der Meßergebnisse nie von besonderer Bedeutung gewesen, als sie sich bei der logarithmischen Darstellung unschwer zu erkennen geben. Der Grund hierfür liegt in dem verhältnismäßig großen Anstieg der Galvanometerkurve (ca. $5-10 \mathrm{~mm} / \mathrm{min}$ ). Demgegenüber machten sich die systematischen Abweichungen bei der Beobachtung der Nachperiode $\lambda_{5}$ ge- 
legentlich sehr störend bemerkbar, da aus experimentellen Gründen der Temperaturunterschied zwischen Thermostat und Kalorimeter in der Nachperiode verhältnismäßig klein war. Daraus folgt eine geringe Steigung der Galvanometerkurve, die teilweise in der Größenordnung der unsystematischen Streuung lag. Die Nachperioden ließen sich deshalb durch ein Bündel logarithmischer Geraden mit teilweise recht unterschiedlicher Neigung darstellen, ohne daß systematische Abweichungen der Meßpunkte zu erkennen gewesen wären. Man kann die Genauigkeit der Extrapolation in diesem Fall allerdings dadurch vergrößern, daß man einmal alle Werte der ersten 7-8 min entsprechend den allgemeinen Erfahrungen unberücksichtigt läßt und für die weiteren Werte eine logarithmische Gerade sucht, deren Steigung möglichst genau gleich der Steigung der Hauptperiode ist (gleiche Abklingkonstante in beiden Fällen). Es wird geschätzt, daß die Fehler in der Extrapolation auch in ungünstigen Fällen $\pm 3 \%$ nicht übersteigen, jedoch sind sie immer noch groß gegenüber einem Fehler von $\pm 0,5$ bis $1 \%$ bei steilen Kurven.

Gegenüber den Extrapolationsfehlern sind alle übrigen Fehler klein. Die Bestimmung der Heizenergie erfolgt mit mindestens $\pm 2 \%$, wenn man folgende Einzelfehler zugrunde legt: Zeitmessung $\pm 0,5^{0} \%$, Widerstandsmessung $\pm 0,2^{0} \%$ und Stromstärkemessung $\pm \mathbf{0 , 5 \%} \%$. Die potentielle Energie der Zertrümmerungsvorrichtung wurde auf etwa $5 \%$ bestimmt. Da sie jedoch nur 2,5\% der Meßwerte beträgt, bleibt im Gesamtergebnis ein Fehler von ca. $1 \%$. Eb . Eenso ist die Konzentrationsbestimmung auch in ungünstigen Fällen auf mindestens $1 \%$ genau.

\section{Meßergebnisse}

Die Meßergebnisse sind in Tab. 1 zusammengestellt und in Abb. 5 zusammen mit den berechneten differentiellen Mischungsenthalpien eingetragen. Die ausgezogene Kurve der integralen molaren Mischungsenthalpie $\Delta \bar{H}$ ist nach der einkonstantigen Gleichung für reguläre Mischungen mit Hilfe der Ausgleichsrechnung nach der Methode der kleinsten Quadrate berechnet worden. Ist $\Delta \bar{H}_{\max }$ die maximale Mischungsenthalpie, so gilt nach Herzfeld und Heitler ${ }^{14}$ :

$$
\Delta \bar{H}=4 \Delta \bar{H}_{\max } x_{1} x_{2} \equiv C x_{1} x_{2} .
$$

${ }^{14}$ K.F. Herzfeld u. W. Heitler, Z. Elektrochem. 31, 536 [1915].
Für die differentiellen Mischungswärmen erhält man :

$$
\Delta H_{1}=C x_{2}{ }^{2}, \Delta H_{2}=C x_{1}{ }^{2} .
$$

\begin{tabular}{|c|c|c|c|c|r|r|r|}
\hline$x_{1}$ & $x_{2}$ & $\Delta_{1}{ }^{*}$ & $\Delta_{2}{ }^{*}$ & $Q$ & $\Delta H^{\prime \prime}$ & \multicolumn{1}{c}{$\Delta H^{\prime}$} & $\Delta \bar{H}$ \\
\hline 87,47 & 12,52 & 44,6 & 34,7 & 4,79 & 6,16 & 6,56 & 14,8 \\
84,03 & 15,97 & 47,3 & 28,8 & 3,84 & 6,32 & 6,96 & 18,8 \\
81,80 & 18,20 & 58,7 & 33,6 & 3,37 & 5,89 & 6,69 & 20,1 \\
75,95 & 24,05 & 73,2 & 42,4 & 5,79 & 10,00 & 10,61 & 26,6 \\
73,75 & 26,25 & 71,1 & 56,2 & 7,67 & 9,70 & 10,41 & 27,2 \\
71,33 & 28,67 & 54,8 & 38,3 & 4,80 & 6,87 & 8,07 & 26,9 \\
71,18 & 28,82 & 67,2 & 51,0 & 6,77 & 8,91 & 9,81 & 27,8 \\
59,01 & 30,99 & 87,6 & 76,0 & 9,71 & 11,19 & 9,73 & 32,4 \\
42,18 & 57,82 & 81,6 & 71,7 & 9,59 & 10,92 & 10,08 & 34,6 \\
41,28 & 58,72 & 78,7 & 66,1 & 8,44 & 10,03 & 9,02 & 33,4 \\
29,39 & 70,61 & 63,7 & 55,9 & 7,68 & 8,75 & 8,57 & 26,9 \\
13,54 & 86,46 & 29,4 & 34,3 & 4,69 & 4,02 & 4,07 & 13,8 \\
& & & &
\end{tabular}

Tab. 1. Integrale Mischungsenthalpien des Systems Benzol-Diphenyl.

$x_{1}=$ Molenbruch des Benzols in \%,

$x_{2}=$ Molenbruch des Diphenyls in $\%$,

$\Delta_{1}{ }^{*}=1$. Ausschlag des Galvanometers in cm, korr.,

$\Delta_{2}{ }^{*}=2$. Ausschlag des Galvanometers in $\mathrm{cm}$, korr.,

$Q^{2}=$ elektrische Energie in cal,

$\Delta H^{\prime \prime}=$ unkorrigierte integrale Mischungsenthalpie in cal,

$\Delta H^{\prime}=$ korrigierte integrale Mischungsenthalpie in cal, $\Delta \bar{H}=$ integrale molare Mischungsenthalpie in cal.

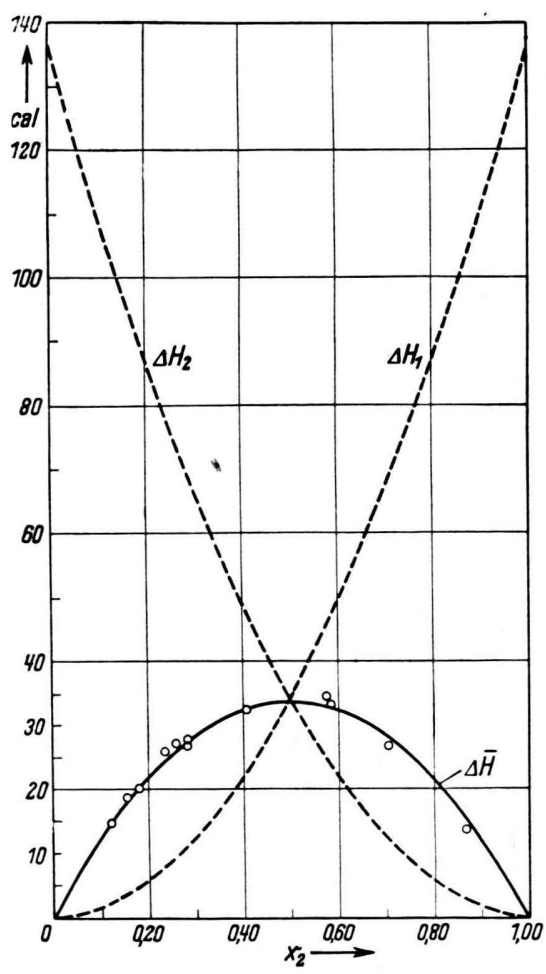

Abb. 5. Integrale und differentielle Mischungsenthalpien des Systems Benzol-Diphenyl bei $71,6^{0} \mathrm{C}$. 
Die Werte der berechneten Konstanten sind: $\Delta \bar{H}_{\max }=34,0$ cal und $C=136,0$ cal. Wieweit Gl. (4) im vorliegenden Fall anwendbar ist, kann nicht einwandfrei geklärt werden. Die Meßpunkte passen sich jedenfalls der Kurve ohne erkennbare systematische Abweichungen an und streuen im Mittel um etwa $2 \%$, was der Fehlergrenze der Messung entspricht.

Aus den differentiellen Mischungswärmen und den von Baxendale bzw. Everett und Mitarb. gemessenen Aktivitätskoeffizienten des Benzols in diesen Mischungen läßt sich die differentielle $\mathrm{Zu}$ satz-Mischungsentropie des Benzols berechnen nach

$$
R T \ln f_{1}=\Delta H_{1}-T \Delta S_{1}{ }^{E} .
$$

Die von Everett angegebenen $\log f_{1}$ Werte bei $65^{\circ} \mathrm{C}$ und $75^{\circ} \mathrm{C}$ sowie die von $\mathrm{Bax} e n d a l e$ angegebenen bei $70^{\circ} \mathrm{C}$ (umgerechnet unter Benutzung der von Everett verwendeten zweiten Virialkoeffizienten des Benzoldampfes) sind in Abb. 6 als Funktion des Molenbruchs $x_{2}$ aufgetragen. Da die Streuungen ziemlich groß sind (die Werte bei $70^{\circ}$ liegen durchweg unterhalb der Werte bei 65 und $75^{\circ}$ ), wurden mittlere Kurven durch die Meßpunkte gelegt, und die zur Berechnung von $\Delta S_{1}{ }^{E}$ notwendigen Zahlenwerte diesen Kurven entnommen. Auf die Umrechnung auf die hier benutzte mittlere Meßtemperatur von $71,6^{\circ} \mathrm{C}$ wurde wegen der Streuung der $\log t$-Werte verzichtet.

Die berechneten Werte für $(-0,4343 / R) \Delta S_{1}{ }^{E}$ sind in Tab. 2 angegeben und in Abb. 7 als Funktion des Molenbruchs aufgetragen. Daneben sind zum Vergleich mit der Theorie die statistisch nach der Näherung von Flory für athermische Mi-

Abb. 6. $\log f_{\text {Benzol }}-x$-Kurven nach Baxendale bzw. Everett u. Mitarbeiter.

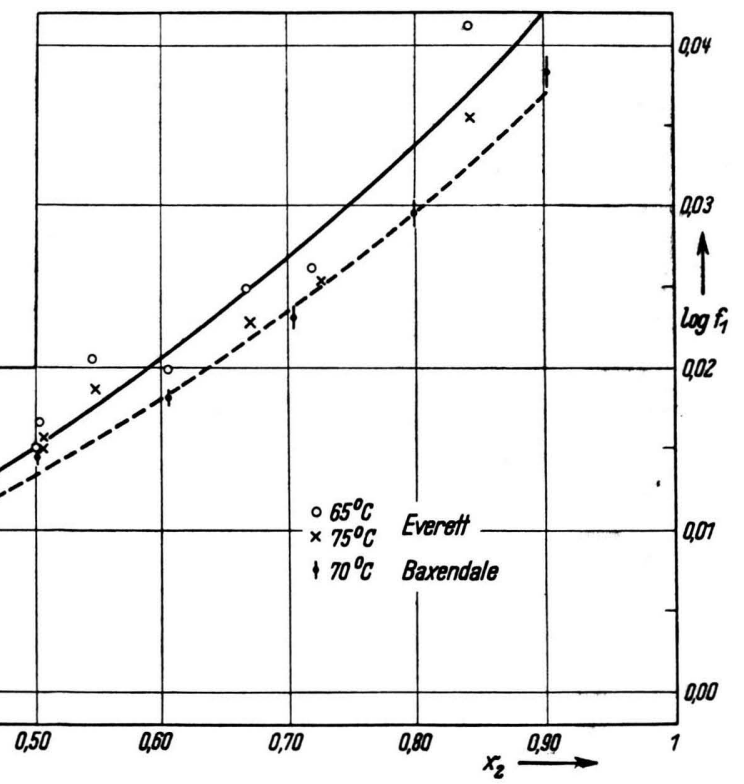

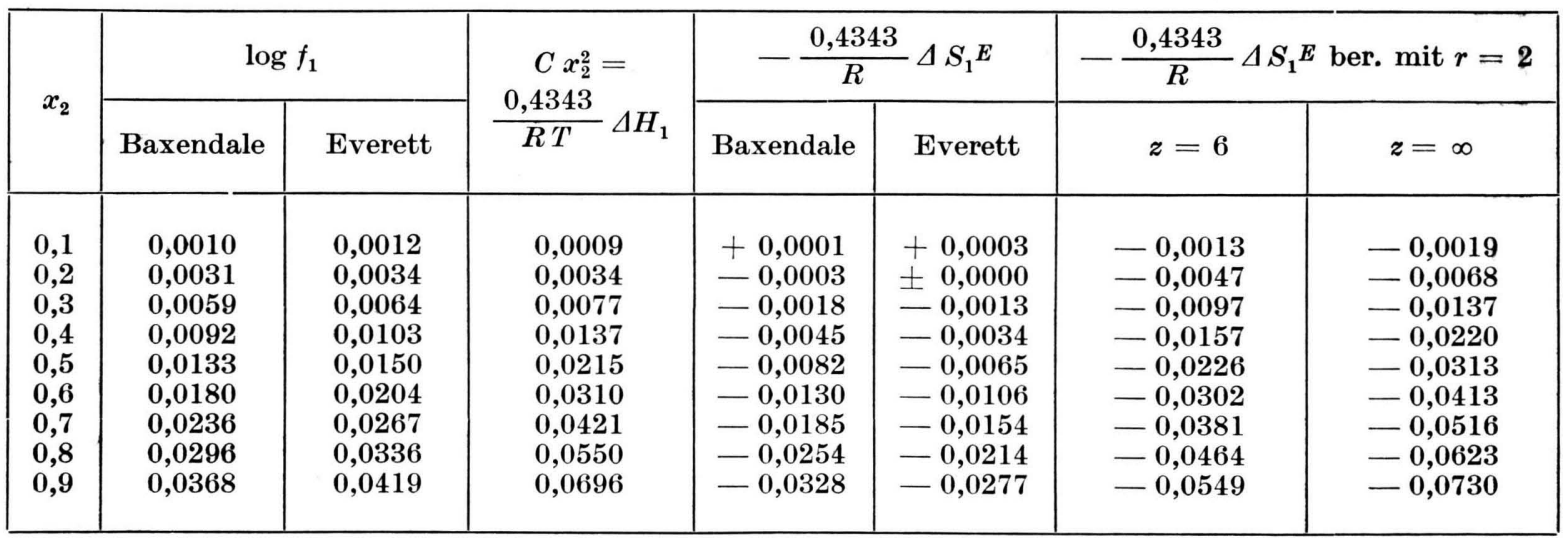

Tab. 2. 
schungen berechneten Werte für $(-0,4343 / R) \Delta S_{1}{ }^{E}$ mit $r=2$ und $z=6$ bzw. $z=\infty$ eingetragen. Der theoretische Bereich ist in Abb. 7 schraffiert. Die Diskrepanz zwischen experimentellen und theoretischen Werten ist also sehr beträchtlich. Erst für $z=2$ würden die Werte etwa übereinstimmen, jedoch ist eine Koordinationszahl 2 im Quasigitter der Flüssigkeit offenbar physikalisch ohne Sinn ${ }^{8}$. Unsere Messungen zeigen ferner, daß die Mischungsenthalpie dieses Systems temperaturab- scheinen die in der Theorie steckenden Annahmen über das Gittermodell bei höheren Temperaturen nicht mehr zuzutreffen, weil hier das freie Volumen infolge der Auflockerung der Flüssigkeit bereits merklichen Einfluß gewinnt, so daß die Diskontinuitäten des Gitters nicht mehr vernachlässigbar sind; andererseits werden durch die Theorie die zweifellos vorhandenen Orientierungseffekte in den reinen Flüssigkeiten ${ }^{16}$, die durch die Mischung mehr oder weniger gestört werden müssen, nicht

Abb. 7 . Differentielle
Zusatz-Mischungsen-
tropie des Benzols im
System Benzol-
Diphenyl bei $71,6^{0} \mathrm{C}$.

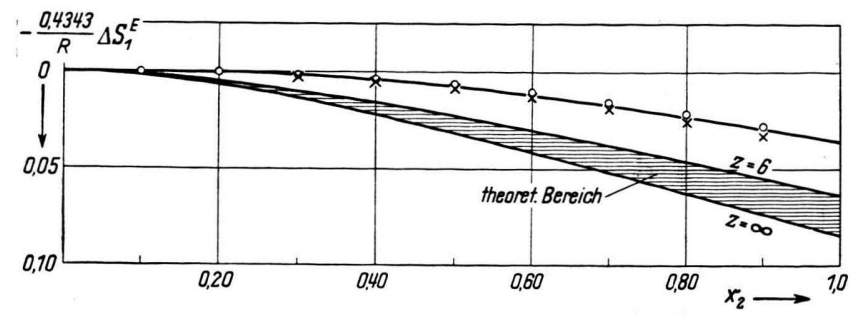

hängig ist, wie auch Tompa ${ }^{8,}{ }^{15}$ vor kurzem festgestellt hat. Bei $25^{\circ} \mathrm{C}$ beträgt die integrale maximale Mischungsenthalpie rund 59 cal. Die Verhältnisse liegen also auch bei diesem System ähnlich wie bei den Paraffinen.

Eine Temperaturabhängigkeit der Mischungsenthalpie bedingt aber auch eine Temperaturabhängigkeit der Zusatzmischungsentropie, die von der Theorie der athermischen Mischungen nicht erklärt werden kann.

Für eine Deutung der Diskrepanz zwischen Theorie und Experiment sind wohl im wesentlichen zwei Möglichkeiten zu diskutieren: Einerseits

berücksichtigt. Diese Ordnungszustände sind natürlich auch temperaturabhängig. Wieviel die eine oder die andere Möglichkeit zu der Größe der beobachteten Abweichungen beiträgt, wird sich erst an Hand eines sehr viel größeren experimentellen Materials entscheiden lassen.

Der Deutschen Forschungsgemeinschaft sowie dem Fonds der Chemie sind wir für die Unterstützung dieser Arbeit zu größtem Dank verpflichtet.

15 H. Tompa, J. chem. Physics 16, 292 [1948].

${ }^{16}$ Vgl. etwa W.C. Pierce, J. chem. Physics 5, 717 [1937]; S.H.de Boer, Trans. Faraday Soc. 32, 10 [1936]; A. Münster, Z. physik. Chem. 196, 106 [1950]. 